\title{
The use of TPA in combination with alcohol in the treatment of the recurrent complex hydrocele
}

\author{
Michael J. Metcalfe, MD; Rebecca J. Spouge, ${ }^{\dagger}$ David J. Spouge, MD; ${ }^{*}$ Chris C. Hoag, MD*
}

*Department of Urologic Sciences, University of British Columbia, Vancouver, BC; †Undergraduate Department of Anatomy and Cellular Biology, McGill University, Montreal, QC; $\S$ Medical Imaging Department, Lions Gate Hospital, Vancouver, BC; *Department of Urology, Lions Gate Hospital, Vancouver, BC

Cite as: Can Urol Assoc J 2014;8(5-6):e445-8. http://dx.doi.org/10.5489/cuaj.1938 Published online June 19, 2014.

\section{Abstract}

A hydrocele is an abnormal collection of serous fluid in the space between the parietal and visceral layers of the tunica vaginalis. Hydrocele is the most common cause of painless scrotal swelling which affects about $1 \%$ of men. Generally, adult hydroceles are idiopathic in origin; however, inguinal surgery, varicocelectomy, infection, trauma and a patent processus vaginalis are each associated with the subsequent development of a hydrocele. Surgical removal of hydroceles is the gold standard of care. However, multiple cases have reported high success rates (ranging from $85 \%$ to $96 \%$ ) using a combination of aspiration and sclerotherapy. We present a case of a patient with recurring complex hydrocele and effective treatment utilizing a combination of thrombolytic therapy, catheter drainage and subsequent alcohol ablation.

\section{Introduction}

A hydrocele is characterized by an abnormal collection of serous fluid in the space between the parietal and visceral layers of the tunica vaginalis. Hydrocele is the most common cause of painless scrotal swelling, affecting about $1 \%$ of men mostly over the age of $40 .{ }^{1}$ While the standard scrotum has been reported to contain 2 to $3 \mathrm{~mL}$ of serous fluid located between the tunical layers, the volume at which the hydrocele is classified is variable. Generally, adult hydroceles are idiopathic in origin; however, varicocelectomy, inguinal surgery, infection, trauma and a patent processus vaginalis are associated with the subsequent development of a hydrocele. ${ }^{2}$

Surgical treatment of hydrocele has been widely accepted as the gold standard of treatment with known, accepted complications including: infection/scrotal abscess formation (3.6\%-9.3\%), treatment failure (persistent swelling or recurrence) $(6 \%-9.3 \%)$, hematoma $(5 \%)$, damage to the epi- didymis and/or vas deferens (3\%), injury to the scrotal contents including the testicle $(2 \%)$, and chronic pain $(0.6 \%) .{ }^{3,4}$ Alternative non-invasive treatments to hydrocelectomy have been utilized. Sclerotherapy with a variety of agents (including phenol, polidocanol, and ethanolamine oleate) and aspiration have been well-documented as safe and effective with success ranging from $58 \%$ to $98 \% .^{5,6}$ We present a patient with recurrent complex hydrocele and a novel percutaneous treatment using a combination of thrombolytic therapy and subsequent alcohol ablation post-hydrocelectomy that demonstrated a complete resolution.

\section{Case report}

A 53-year-old male presented with a longstanding symptomatic left hydrocele and wanted it repaired. Ultrasound revealed a moderate left and minimal right simple hydrocele with normal epididymal and testicular structures and moderate left hemiscrotum subcutaneous soft tissue thickening (Fig. 1).

Surgical repair was undertaken with midline incision and dissection of the hydrocele sac. Delivery of the sac was performed with subsequent incision and drainage. The excess sac was excised and the edges sutured with 3-0 monocryl using a Jaboulay technique. Due to concern about torsion in the redundant scrotal sac, the testicle was pexed from the edges of the hydrocele sac to the dartos fascia with two 2-0 Vicryl sutures. The dartos was closed with 2-0 Vicryl and the skin closed with 3-0 Vicryl Rapid.

Within a week of the surgery, the patient described gradual return of scrotal swelling. About 3 months after the surgery, the patient remained symptomatic and underwent ultrasound examination. The ultrasound revealed a recurrent moderate hydrocele with minimal homogeneous internal echoes. No septations were evident. Four months later the patient experienced a sudden increase in scrotal size. The ultrasound revealed echogenic fluid with occasional septations. 


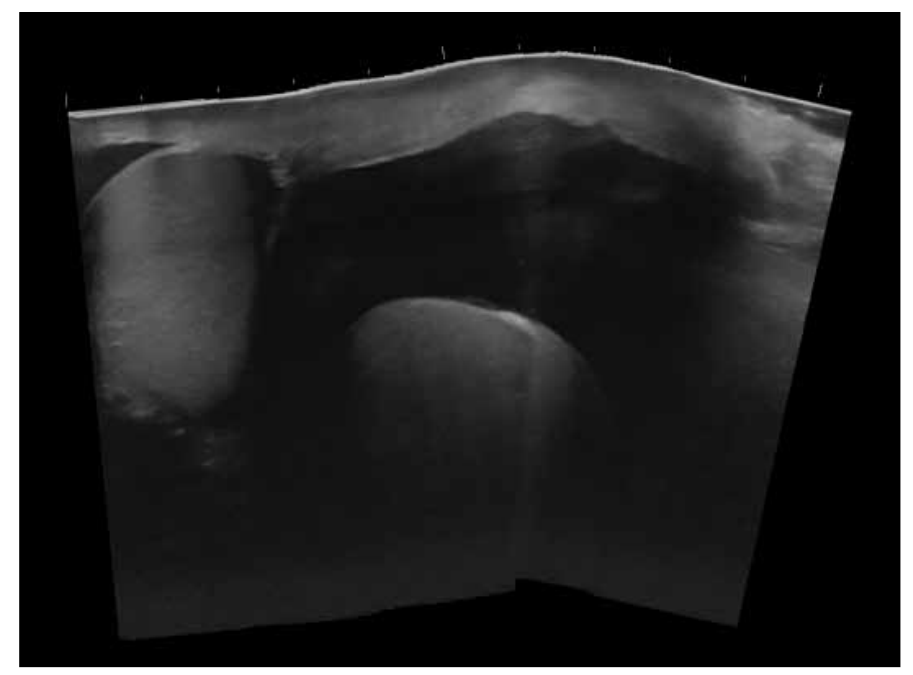

Fig. 1. Transverse extended field of view scrotal sonogram at time of patient presentation revealing large left and minor right simple hydroceles with moderate left hemiscrotum skin thickening.

A second hydrocelectomy surgery was performed 1 week following the ultrasound. A midline incision was made over prior incision. Adhesions were dissected and cautery was used to dissect and deliver the sac. Serosanguinous fluid was drained and subsequently the sac was excised and the edges were cauterized and oversewn.

Within a week of the hydrocele repair, rapid reaccumulation occurred. Four months later, a third surgery was performed (11 months after the the first operation). The possibility of a persistent patent processus vaginalis was assessed at the time of repeat hydrocelectomy with laparoscopic visualization of the inguinal ring with methylene blue instillation into left hemiscrotum. This revealed no definite communication, but dimpling of the internal ring was

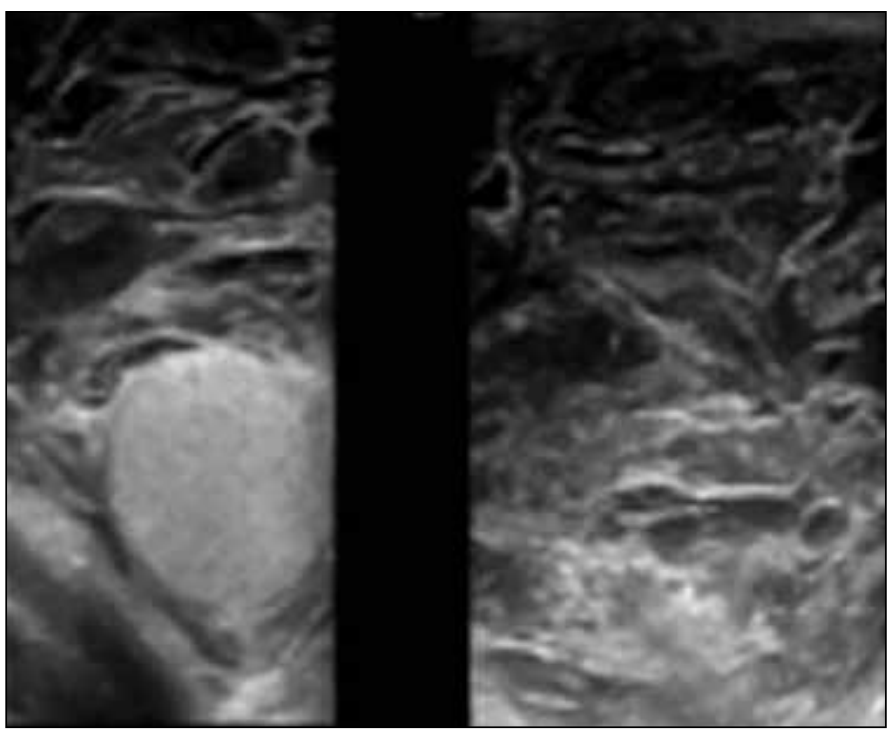

Fig. 2. Transverse scrotal sonograms after 3 prior surgical repairs indicating extremely complex hydrocele with multiple thick septations. visualized and consequently the internal ring was ligated. The pseudosac was re-excised in a similar manner to the previous procedure. A quarter inch penrose drain was then left in situ.

Symptomatic reaccumulation occurred within 1 week of the operation. At this time, interventional radiology was consulted with regard to possible sclerotherapy of recurrent hydrocele. The ultrasound at the time of consultation revealed a grossly septated large hydrocele (Fig. 2). A 5-Fr Yueh catheter was inserted via ultrasound guidance and about 30 cc Omnipaque 300 was instilled and subsequently aspirated. A patent processus vaginalis was excluded radiographically.

The patient was informed that the fluid collection was extremely complex and septated and that the chance of success was modest. As well, the patient was informed that sclerotherapy for a hydrocele that had previously been operated on 3 times before had not been, to our knowledge, previously described. The multiseptated appearance suggested likely failure of a simple procedure involving drainage and sclerotherapy. Given the only option being recurrent surgery, the patient was counselled regarding the trial use of thrombolytics with or without associated sclerotherapy. The patient indicated a desire for a nonoperative procedure, even without the documentation of historical success.

A 10-Fr pigtail catheter was subsequently inserted into the left hemiscrotum following exchange of the Yueh catheter over a $0.03815 \mathrm{~J}$ tip guidewire and $50 \mathrm{cc}$ serosanguinous of fluid was drained. Contrast was instilled to ensure there was no communication with the peritoneum (Fig. 3). In total, $6 \mathrm{mg}$ of TPA (alteplase) was diluted in $20 \mathrm{cc}$ normal saline, instilled and left overnight.

The following day, the catheter was aspirated and an ultrasound revealed near resolution of the hydrocele, with the exception of a 3.7-cm non-communicating inferior collection. A second 8-Fr catheter was subsequently inserted into the inferior collection and the serosanguinous fluid completely aspirated. Catheters were then closed and left for 5 days to assess for reaccumulation or resolution. It was felt that reaccumulation would indicate the need for alcohol sclerosis.

Unfortunately reaccumulation of a complex hydrocele did occur. Again, $6 \mathrm{mg}$ of TPA in $20 \mathrm{cc}$ normal saline was instilled and left overnight with the catheter capped. The patient returned the next day with complete lysis of septations and the fluid was subsequently completely aspirated through the indwelling catheter. In total, 20 cc $0.5 \%$ marcaine was instilled for 30 minutes and then drained. Moreover, $30 \mathrm{cc}$ of dehydrated ethanol was instilled and left for 2 hours while the patient was repositioned. The alcohol was then aspirated and the catheter was removed.

One week later at the office visit to the referring urologist, the patient had dramatically improved. Minimal discomfort 
TPA with alcohol to treat recurrent complex hydrocele

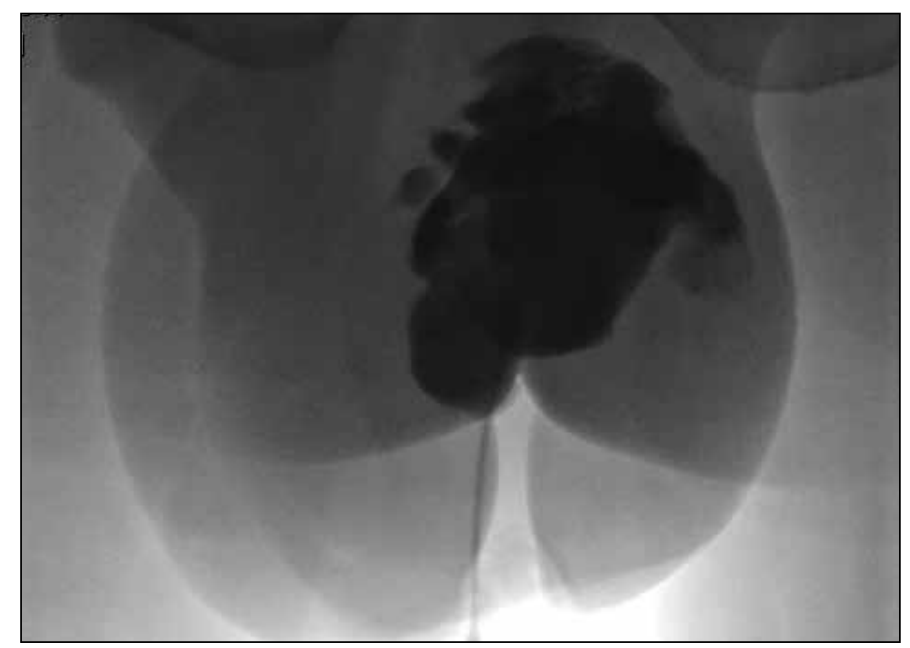

Fig. 3. Fluoroscopic asessment with insertion of contrast revealing loculation and compartmentalization of complex hydrocele and the absence of peritoneal communication.

occurred following sclerosis. Only mild edema and induration was evident with one region of fluctuance suggesting a small residual pocket. Six weeks after the procedure, an office visit revealed no clinical hydrocele. Some residual scrotal induration was present, but significantly improved. Seven months later, a follow-up ultrasound revealed no evidence of hydrocele recurrence. The patient was asymptomatic. Minor subcutaneous soft tissue thickening was evident on ultrasound and had diminished compared with the preprocedure exam (Fig. 4).

\section{Discussion}

Hydroceles are abnormal collections of serous fluid within the potential space of the tunica vaginalis occurring in $1 \%$ of males. ${ }^{7}$ Acquired idiopathic hydroceles are the most common cause of enlarged scrotal size in adults and are thought to originate from an imbalance in the production of serous fluid and lymphatic drainage. Although a benign process, the enlarged scrotum provides significant discomfort and motivates most patients to seek correction. Surgical removal of the hydrocele has been well-documented as the accepted standard of care.

In this case, the surgeon went above standard measures to ensure complete resection of the hydrocele and appropriate closure of the inguinal ring. However, this hydrocele was clearly refractory. The issue was likely due to an abnormal cell layer within the testicle that, despite trauma from 3 operations and subsequent adhesions, remained very active in secretion. Therefore, non-surgical options were explored.

Several nonsurgical treatments in the management of hydroceles have been well-documented. Aspiration both singularly and accompanied by follow-up sclerotherapy using various sclerosing agents have been reported with a generally effective response. A review of the literature reports

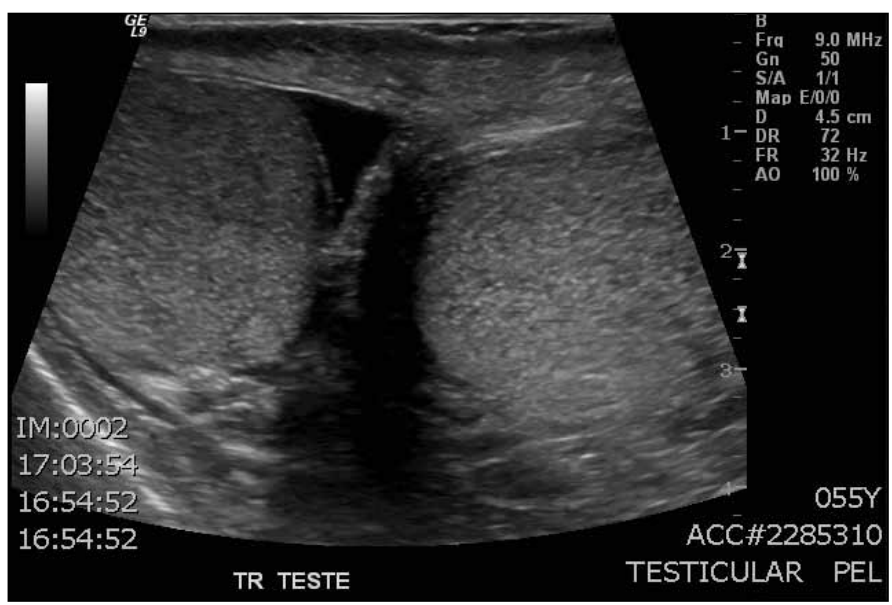

Fig. 4. Transverse scrotal sonogram at followup revealing complete resolution of left hydrocele and persisting left hemiscrotal skin thickening.

a variety of effective agents including: polidcoconol, phenol, tetracycline ethanolamine oleate, STS and alcohol. Each agent reports a cure rate ranging from $85 \%$ to $97 \%$, with complication rates of up to $50 \% .^{8-12}$ Alcohol is a sclerosing agent that has a chemical irritant effect on the contacting tissue. The necrosis produced by alcohol injection is secondary to cellular dehydration, cell membrane lysis, and protein denaturation. Additionally, it induces vascular thrombosis and subsequent tissue ischemia. ${ }^{13}$ Alcohol, therefore, was an ideal pharmacotherapeutic option for hydrocelectomy complicated by recurring complex hydrocele formation.

To our knowledge, the use of TPA following hydrocelectomy has not been previously. However, given the multiple septations within the hydrocele it was necessary to break them down to maximize the effect of the alcohol. TPA is similar to the plasminogen activator in blood, does not result in antibody activation, and has greater fibrin specificity than most other thrombolytic drugs. ${ }^{14}$ While TPA is generally an accepted form of treatment for intravascular thrombolysis, it has also been used in the minimally invasive evacuation of intracerebral and intraventricular hemorrhage, the treatment of undrained traumatic hemothorax, and in the decompression of retroperitoneal hematoma following abdominal aortic aneurysm rupture as an adjunct to endovascular repair. ${ }^{15-17}$ TPA was effective in breaking down the septations and allowing for the sclerosing agent to contact most of the inner lining of the hydrocele. There was no hemorrhage associated with its use in this case, although we recognize the potential.

\section{Conclusion}

We describe the successful use of a combination of TPA for thrombolysis and minimally invasive evacuation of a recurrent, complex hydrocele with subsequent sclerotherapy with alcohol. 
Metcalfe et al.

Competing interests: competing financial or personal interests.

This paper has been peer-reviewed.

\section{References}

1. Wallace AF. Aetiology of the idiopathic hydrocele. Br J Uro 1963;32:79-96. http://dx.doi.org/10.1111/ j.1464-410X.1960.tb00031.x

2. Cimador $M$, Castagnetti M, De Grazia E. Management of hydrocele in adolescent patients. Nat Rev Urol 2010;7:379-85.

3. Kiddoo DA, Wollin TA, Mador DR. A population based assessment of complications following outpatient hydrocelectomy and spermatocelectomy. J Urol 2004;171:746-8. http://dx.doi.org/10.1097/01. ju.0000103636.61790.43

4. Swartz MA, Morgan TM, Krieger JN. Complications of scrotal surgery for benign conditions. Urology 2007;69:616. http://dx.doi.org/10.1016/i.urology.2007.01.004

5. Daehlin L, Tonder B, Kapstad L. Comparison of polidocanol and tetracycline in the sclerotherapy of testicular hydrocele and epididymal cys. Br J Urol 1997;80:468-71. http://dx.doi.org/10.1046/i.1464410X.1997.00358.x

6. Tammela TL, Hellstrom PA, Matilla SI, et al. Ethanolamine oleate sclerotherapy for hydroceles and spermatoceles: A survey of 158 patients with ultrasound follow-up. J Urol 1992;147:1551-3.

7. Leung ML, Gooding GA, Williams RD. High-resolution sonography of scrotal contents in asymptomatic subjects. AJR Am J Roentgenol 1984;143:161. http://dx.doi.org/10.2214/air.143.1.161
8. Levine LA, DeWolf WC. Aspiration and tetracycline sclerotherapy of hydroceles. J Uro/ 1988;139:959-60.

9. Shan CJ, Lucon AM, Pagani R, et al. Sclerotherapy of hydroceles and spermatoceles with alcohol: Results and effects on the semen analysis. Int Braz I Urol 2011;37:307-13. http://dx.doi.org/10.1590/ S1677-55382011000300003

10. Miskowiak J, Christensen AB. Treatment of hydrocele testis by injection of tetracycline. Eur Urol 1998; 14:440-1.

11. Agrawal MS, Yadav H, Upadhyay A, et al. Sclerotherapy for hydrocele revisited: A prospective randomised study. Indian J Surg 2009;71:23-8. http://dx.doi.org/10.1007/s12262-009-0006-7

12. Rencken RK, Bornman MS, Reif S, et al. Sclerotherapy for hydroceles. J Urol 1990;143:940-3.

13. Leyendecker JR, Dodd GD. Minimally invasive techniques for the treatment of liver tumors. Semin Liver Dis 2001;21:283-91. http://dx.doi.org/10.1055/s-2001-15345

14. Collen D, Lijnen HR, Todd PA, et al. Tissue-type plasminogen activator. A review of its pharmacology and therapeutic use as a thrombolytic agent. Drugs 1989;38:346-88. http://dx.doi.org/10.2165/00003495198938030-00003

15. Dey M, StadnikA, Awad IA. Thrombolytic evacuation of intracerebral and intraventricular hemorrhage. Curr Cardiol Rep 2012;14:754-60. http://dx.doi.org/10.1007/s1 1886-012-0316-4

16. Kimbrell BJ, Yamzon J, Petrone P, et al. Intrapleural thrombolysis for the management of undrained traumatic hemothorax: A prospective observational study. J Trauma 2001;62:1175-8. http://dx.doi. org/10.1097/TA.0b013e3180500654

17. Hörer T, Skoog P, Pirouzram A, et al. Tissue plasminogen activator-assisted hematoma evacuation to relieve abdominal compartment syndrome after endovascular repair of ruptured abdominal aortic aneurysm. J Endovasc Ther 2012;19:144-8. http://dx.doi.org/10.1583/11-3699.1

Correspondence: Dr. Michael Joseph Metcalfe, Department of Urologic Sciences, University of British Columbia, Vancouver, BC; michaelmetcalfe5@gmail.com 